\title{
Form-finding with Polyhedral Meshes Made Simple
}

\author{
Chengcheng Tang, Xiang Sun, Alexandra Gomes \\ King Abdullah University of Science and Technology (KAUST)
}

\author{
Johannes Wallner
}

TU Graz
Helmut Pottmann
KAUST / TU Wien

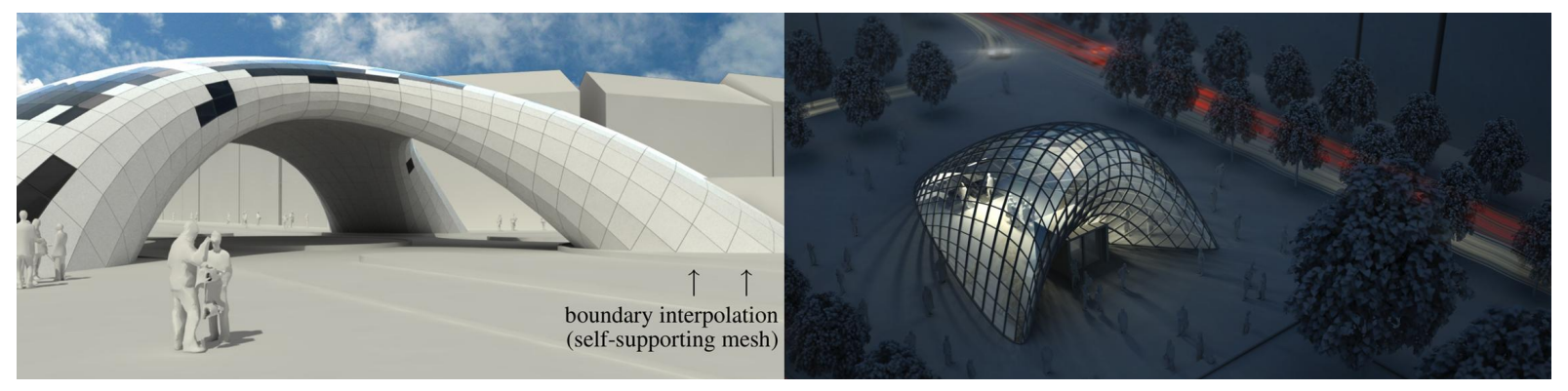

Figure 1: Form-finding with polyhedral meshes for architectural design. We develop an interactive modeling system which can handle geometric properties like planarity of faces, compression-only equilibrium forces in the edges and other properties like constraining boundary vertices to previously specified boundary curves (this constraint is not at all easy to satisfy for quad meshes with planar faces).

\begin{abstract}
We solve the form-finding problem for polyhedral meshes in a way which combines form, function and fabrication; taking care of userspecified constraints like boundary interpolation, planarity of faces, statics, panel size and shape, enclosed volume, and cost. Our main application is the interactive modeling of meshes for architectural and industrial design. Our approach can be described as guided exploration of the constraint space whose algebraic structure is simplified by introducing auxiliary variables and ensuring that constraints are at most quadratic.
\end{abstract}

\section{Introduction and Overview}

Shape modeling systems often provide the user with little support to satisfy constraints implied by function and fabrication of the designed product. Very recently however we see a trend towards novel design tools combining form, function and fabrication. This new integrated approach is a big challenge and obviously more specific to the intended application than classical geometric modeling.

We develop a framework for the interactive modeling of polyhedral surfaces or - more generally - meshes constrained by equalities and inequalities. In particular we handle self-supporting meshes and give the user control over both combinatorics and geometry. The framework goes well beyond meshes for architecture (which we used as our main motivation).

\section{Algorithms and Results}

On the technical level, we solve constraint equations for meshes by a two-step process: Preprocessing (i.e., the choice of variables) makes the constraint equations quadratic as far as possible. The actual computation is done by a Newton-type method, numerical

Permission to make digital or hard copies of part or all of this work for personal or classroom use is granted without fee provided that copies are not made or distributed for commercial advantage and that copies bear this notice and the full citation on the first page. Copyrights for third-party components of this work must be honored. For all other uses, contact the Owner/Author.

Copyright is held by the owner/author(s).

SIGGRAPH 2015 Posters, August 09 - 13, 2015, Los Angeles, CA.

ACM 978-1-4503-3632-1/15/08.

http://dx.doi.org/10.1145/2787626.2787631 problems due to redundant constraints being circumvented by using a fairness energy for regularization.

The main successes of our approach are its speed (it is fast enough to allow for interactive modeling of constrained meshes without GPU implementation), and also the easy way to deal with problems which so far have been considered difficult: handling force equilibrium and force constraints in meshes which are not locally height fields, and manipulating both combinatorics and shape. The authors are not aware of previous work in this area which for instance is able to combine statics with planarity constraints. We are confident that the method of guided projection onto constraint manifolds presented in this paper will find applications completely unrelated to architecture, and even unrelated to meshes.

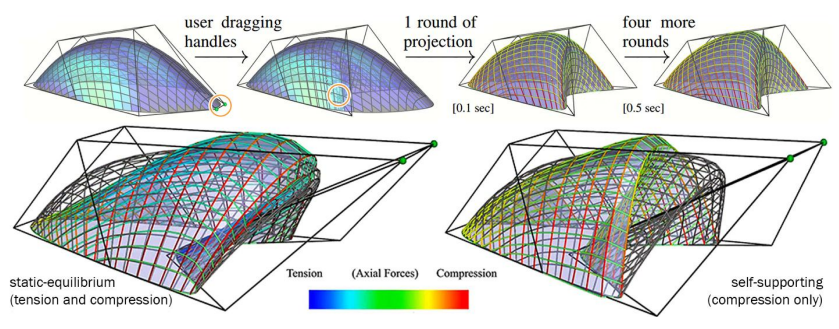

Figure 2: Screen shots of interactive design with planarity and statics based on subdivision modeling, coupled with guided projection onto the constraint manifold. Note that our method provides perfect user-controlled boundary interpolation: Partly the boundary is a mesh polyline, partly the boundary consists of diagonals. Please also note that in contrast to the mesh in static equilibrium with both tension and compression (bottom-left), self-supporting allows only compression, and prevents the top of the structure further leaning forward (bottom-right).

\section{References}

C. Tang, X. Sun, A. Gomes, J. Wallner, and H. Pottmann. Formfinding with polyhedral meshes made simple. ACM Trans. Graphics, 33(4), 2014. Proc. SIGGRAPH. 